\title{
EACH HYPERINVARIANT SUBSPACE FOR MULTIPLICATION OPERATOR IS SPECTRAL
}

\author{
HUANG SENZHONG
}

(Communicated by Paul S. Muhly)

\begin{abstract}
We consider multiplication operators on general separable complex $L^{p}$-spaces, for $1 \leq p<+\infty$, and obtain the result announced in the title. Moreover, a result of Douglas and Pearcy on normal operators is given an alternate proof.
\end{abstract}

In this paper, our notation is standard. We refer the reader to [1] and [4]. We consider only complex spaces $L^{p}(X, \mathscr{A}, \mu, \mathbb{C})$ for $1 \leq p \leq+\infty$. We also simply denote $L^{p}(X, \mathscr{A}, \mu, \mathbb{C})$ by $L^{p}(X, \mathscr{A}, \mu)$ or $L^{p}(X, \mu)$ in the circumstances where there is no confusion.

Let $\phi \in L^{\infty}(X, \mu)$. The multiplication operator corresponding to $\phi$ is the bounded operator $M_{\phi}$ on $L^{p}(X, \mu)$ defined by $\left(M_{\phi} f\right)(x)=\phi(x) f(x)$ for all $f \in L^{p}(X, \mu)$. For a Borel set $S$ of $\mathbb{C}$, define $E^{c}(S)=M_{1_{S} \circ \phi}$, where by $1_{S}$ we always denote the characteristic function corresponding to a set $S$. Following Dunford [3], $E^{c}(\cdot)$ is a spectral measure which makes $M_{\phi}$ a spectral operators. For a spectral operator, there is the following remarkable theorem of Fuglede and Dunford (its proof can be found in [5]).

F-D Theorem. If $A$ is a spectral operator with spectral measure $E(\cdot)$ and if $A B=B A$, then $B E(S)=E(S) B$ for all Borel sets $S$.

As a result of this theorem, we have

Corollary. Let $E(\cdot)$ be any spectral measure for $M_{\phi}$. If $\mathscr{M}$ is the range of $E(S)$ for some Borel set $S$, then $\mathscr{M}$ is a hyperinvariant subspace for $M_{\phi}$. Thus each nonscalar multiplication operator has a nontrivial hyperinvariant subspace.

Proof. Trivial.

From this corollary we see that the range of $E^{c}(S)$ for each Borel set $S$ is a hyperinvariant subspace for $M_{\phi}$. We will next see that each hyperinvariant subspace for $M_{\phi}$ can be written in this form whenever $M_{\phi}$ is defined on a separable space. Because of this, by $E^{c}(\cdot)$ we always mean this special spectral

Received by the editors November 24, 1988.

1980 Mathematics Subject Classification (1985 Revision). Primary 47A15, 47B37.

Key words and phrases. Hyperinvariant subspace. 
measure defined as at the beginning. Moreover, by a subspace we always mean a linear subspace and we call a closed subspace $\mathscr{M}$ of $L^{p}(X, \mu)$ a spectral subspace if $\mathscr{M}=E^{\mathcal{c}}(S) L^{p}(X, \mu)$ for some Borel set of $\mathbb{C}$. We also denote the algebra of all multiplication operators on $L^{p}(X, \mu)$ by $\mathscr{L}^{\infty}$, i.e.

$$
\mathscr{L}^{\infty}=\left\{M_{\phi}: \phi \in L^{\infty}(X, \mu)\right\}
$$

Let us start with a converse of the F-D Theorem for multiplication operators.

Lemma 1. If $B$ commutes with all $E^{c}(S)$, then $B$ commutes with $M_{\phi}$.

Proof. This is trivial by the definition of the spectral integral.

Now we pass to the structure of hyperinvariant subspaces for multiplication operators.

Lemma 2. Let $\mathscr{M}$ be a closed separable subspace of $L^{p}(X, \mathscr{A}, \mu)$ for $1 \leq p<$ $+\infty$. If $\mathscr{M}$ is invariant for $\mathscr{L}^{\infty}$, i.e. $M_{\phi} \mathscr{M} \subseteq \mathscr{M}$ for all $M_{\phi} \in \mathscr{L}^{\infty}$, then there exists some $A \in \mathscr{A}$ such that $\mathscr{M}=1_{A} \cdot L^{p}(X, \mathscr{A}, \mu)$.

Proof. Since $\mathscr{M}$ is separable, a theorem of Ando (see [4], page 152, Lemma 1) implies that there exists an $f \in \mathscr{M}$ with maximal support $A$ of all functions in $\mathscr{M}$. It follows that $A \in \mathscr{A}$ and $\mathscr{M} \subseteq 1_{A} \cdot L^{p}(X, \mu)$. We must show that $\mathscr{M}=1_{A} \cdot L^{p}(X, \mu)$. To this end, assume $A \supseteq B \in \mathscr{A}$ with $\mu(B)<+\infty$. Let

$$
A_{n}=\left\{x \in X: \frac{1}{n} \leq|f(x)| \leq n\right\} \text { for all } n \geq 1 .
$$

Put $\phi_{n}=f^{-1} \cdot 1_{A} \cdot 1_{B}$. Then $\phi_{n} \in L^{\infty}(X, \mu)$; and hence $1_{B \cap \dot{A}_{n}}=\phi_{n} \cdot f \in \mathscr{M}$. We have that $\mu\left(B \backslash B \cap A_{n}\right) \rightarrow 0$ as $n \rightarrow \infty$. Since $\mathscr{M}$ is closed, this implies that $1_{B} \in \mathscr{M}$. And thus $1_{B} \cdot L^{\infty}(X, \mu) \subseteq \mathscr{M}$ for all $A \supseteq B \in \mathscr{A}$ with $\mu(B)<+\infty$. It is now routine to check that $\mathscr{M}=1_{A} \cdot L^{p}(X, \mu)$ since $\mathscr{M}$ is closed and since the span of subspaces $1_{B} \cdot L^{\infty}(X, \mu)$ for $A \supseteq B \in \mathscr{A}$ with $\mu(B)<+\infty$ is dense in $1_{A} \cdot L^{p}(X, \mu)$. This completes the proof.

To continue, we need one more notion. Let $(X, \mathscr{A}, \mu)$ be a finite measure space and let $\phi \in L^{\infty}(X, \mathscr{A}, \mu)$. Let $\mathscr{A}_{0}=\left\{\phi^{-1}(S): S\right.$ a Borel set of $\left.\mathbb{C}\right\}$. It is easily seen that $\mathscr{A}_{0}$ is also a sub-sigma-algebra of $\mathscr{A}$. We regard $\left(X, \mathscr{A}_{0}, \mu\right)$ as a finite measure space. Let $A \in \mathscr{A}$ be given. By the well-known RadonNikodym theorem we may define the conditional expectation operator $E_{A}$, for the measure $\mu$ relative to $\mathscr{A}_{0} \cdot E_{A}$ is uniquely determined by the equation

$$
\int_{B} h \cdot 1_{A} d \mu=\int_{B}\left(E_{A} h\right) d \mu \quad\left(B \in \mathscr{A}_{0}\right)
$$

for $h \in L^{1}(X, \mathscr{A}, \mu)$ and by the condition that $E_{A} h$ is $\mathscr{A}_{0}$-measurable. This class of operators $\left\{E_{A}: A \in \mathscr{A}\right\}$ has the following interesting properties.

Lemma 3. (1) Each $E_{A}$ is a positive linear operator on $L^{p}(X, \mu), 1 \leq p \leq \infty$ with $\left\|E_{A}\right\| \leq 1$. In particular, $0 \leq E_{A}(h) \leq\|h\|_{\infty}$ for all $h \in L^{\infty}(X, \mu)$ with $0 \leq h$.

(2) Each $E_{A}$ commutes with $M_{\phi}$, i.e. $E_{A} M_{\phi}=M_{\phi} E_{A}$. 
Proof. (1) We may write $E_{A}=E_{X} \circ M_{1_{A}}$, where $E_{X}$ is the usual conditional expectation operator determined by the sub-sigma-algebra $\mathscr{A}_{0}$ (cf. [1]). Since both $E_{X}$ and $M_{1_{A}}$ are positive contractions on each $L^{p}(X, \mu), 1 \leq p \leq \infty$, as is well known, (1) follows.

(2) By Lemma 1, it suffices to show that $E_{A}$ commutes with all $E^{c}(S)$ for Borel sets $S$ of $\mathbb{C}$. Fix such an $S$. Then $E^{\mathcal{C}}(S)=M_{1_{\phi}-1_{(S)}}$ and $\phi^{-1}(S) \in \mathscr{A}_{0}$. By the definition of $E_{A}$, for all $B \in \mathscr{A}_{0}$,

$$
\begin{aligned}
\int_{B} E^{c}(S)\left(E_{A} h\right) d \mu & =\int_{B \cap \phi^{-1}(S)}\left(E_{A} h\right) d \mu=\int_{B \cap \phi^{-1}(S)} h \cdot 1_{A} d \mu \\
& =\int_{B}\left(E^{c}(S) h\right) \cdot 1_{A} d \mu=\int_{B} E_{A}\left(E^{c}(S) h\right) d \mu,
\end{aligned}
$$

for all $h \in L^{1}(X, \mathscr{A}, \mu)$. Since both $E^{c}(S)\left(E_{A} h\right)$ and $E_{A}\left(E^{c}(S) h\right)$ are $\mathscr{A}_{0}$ measurable, we conclude from the above that $E_{A} E^{c}(S)=E^{c}(S) E_{A}$. This finishes the proof.

Lemma 4. Let $(X, \mathscr{A}, \mu)$ be a finite measure space and $1 \leq p \leq+\infty$. Let $\phi \in$ $L^{\infty}(X, \mu)$ and $\mathscr{M}=1_{A} \cdot L^{p}(X, \mu)$ for some $A \in \mathscr{A}$. Then $\mathscr{M}$ is hyperinvariant for $M_{\phi}$ iff $A=\phi^{-1}(S)$ for some Borel set $S$, i.e. $\mathscr{M}$ is a spectral subspace.

Proof. Assume that $\mathscr{M}=1_{A} \cdot L^{p}(X, \mu)$ is hyperinvariant for $M_{\phi}$. By Lemma 3 again, $E_{A}$ is a bounded operator which commutes with $M_{\phi}$. So $E_{A}(\mathscr{M}) \subseteq \mathscr{M}$. In particular, there exists $f_{0} \in L^{p}(X, \mu)$ such that $E_{A}\left(1_{A}\right)=1_{A} \cdot f_{0}$. Let $f_{1}=E_{A}\left(1_{A}\right)$. By Lemma 3, again $0 \leq f_{1} \leq 1$ a.e. Note that

$$
\int_{X}\left(1-f_{1}\right) \cdot 1_{A} d \mu=\int_{X} 1_{A} d \mu-\int_{X}\left(E_{A} 1_{A}\right) d \mu=0,
$$

and so $\left(1-f_{1}\right) \cdot 1_{A}=0$ a.e. Hence $1_{A}=1_{A} \cdot f_{1}=E_{A}\left(1_{A}\right)$ is $\mathscr{A}_{0}$-measurable. This implies that $A=\phi^{-1}(S)$ for some Borel set $S$. The converse is the corollary to the F-D Theorem.

Lemma 5. Let $(X, \mu)$ be a finite measure space and $1 \leq p<+\infty$. Let $\phi \in$ $L^{\infty}(X, \mu)$ and let $M_{\phi}$ be the multiplication operator on $L^{p}(X, \mu)$. Then a closed separable subspace $\mathscr{M}$ of $L^{p}(X, \mu)$ is hyperinvariant for $M_{\phi}$ iff $\mathscr{M}$ is spectral, i.e. $\mathscr{M}=E^{c}(S) L^{p}(X, \mu)$ for some Borel set $S$.

Proof. Use Lemma 2 and Lemma 4 while observing that each element of $\mathscr{L}^{\infty}$ commutes with $M_{\phi}$.

For a Banach space $Z$ and a bounded linear operator $T$ on $Z$, let $\operatorname{comt}(T)$ be the commutant of $T$, i.e. the set of all bounded linear operators on $Z$ which commute with $T$. If $V: Z \rightarrow Y$ is an onto-isomorphism, then $\operatorname{comt}\left(V T V^{-1}\right)$ $=V \cdot \operatorname{comt}(T) \cdot V^{-1}$.

Next, we state our main theorem. 
Theorem 1. Let $1 \leq p<+\infty$ and $(X, \mu)$ be a measure space such that the space $L^{p}(X, \mu)$ is separable. Let $\phi \in L^{\infty}(X, \mu)$ and $M_{\phi}$ be the multiplication operator on $L^{p}(X, \mu)$. Then a closed subspace $\mathscr{M}$ of $L^{p}(X, \mu)$ is hyperinvariant for $M_{\phi}$ iff $\mathscr{M}$ is spectral, i.e. $\mathscr{M}=E^{c}(S) L^{p}(X, \mu)$ for some Borel set $S$.

Proof. Since $L^{p}(X, \mu)$ is separable, by the same theorem of Ando used in the proof of Lemma 2 above, one can easily build a finite measure $\nu$ on $X$ such that $\nu$ is equivalent to $\mu$. Define $V: L^{p}(X, \mu) \rightarrow L^{p}(X, \nu)$ by

$$
V f=f \cdot\left(\frac{d \mu}{d \nu}\right)^{1 / p} \quad \text { for all } f \in L^{p}(X, \mu) .
$$

Then $V$ is an onto-isometric isomorphism such that $V M_{\phi} V^{-1}$ is the multiplication operator $M_{\phi}$ on $L^{p}(X, \nu)$. From the previous remark, we immediately obtain that $\mathscr{M}$ is hyperinvariant for $M_{\phi}$ on $L^{p}(X, \mu)$ iff $V \mathscr{M}$ is also hyperinvariant for $M_{\phi}$ on $L^{p}(X, \nu)$. Use Lemma 5 while observing the definition of $V$, the latter assertion is equivalent to

$$
\mathscr{M}=E^{c}(S) L^{p}(X, \mu) \quad \text { for some Borel set } S .
$$

This completes the proof.

We give two applications of Theorem 1.

Corollary 1. Suppose $1 \leq p \neq 2<+\infty$. Let $L^{p}(X, \mu)$ be separable and let $\phi \in L^{\infty}(X, \mu)$. Let $E^{c}(\cdot)$ be the special spectral measure corresponding to $M_{\phi}$ on the space $L^{p}(X, \mu)$. Then $E^{c}(\cdot)$ is maximal in the sense that if $E(\cdot)$ is another spectral measure for $M_{\phi}$ in the sense of Dunford [3] with contractive projections, then the range of $E(\cdot)$ is contained in that of $E^{c}(\cdot)$.

Proof. Fix a Borel set $S$. Let $\mathscr{M}=E(S) L^{p}(X, \mu)$. By the corollary to the F-D Theorem, $\mathscr{M}$ is hyperinvariant for $M_{\phi}$. Now Theorem 1 implies that $\mathscr{M}=E^{c}\left(S_{1}\right) L^{p}(X, \mu)$ for some Borel set $S_{1}$. It follows that $E(S) E^{c}\left(\mathbb{C} \backslash S_{1}\right)=0$ since $E(S)$ commutes with $E^{c}\left(\mathbb{C} \backslash S_{1}\right)$ by the F-D Theorem. We consider the space $L^{p}(X, \mu)$ as a complex Banach lattice, and we refer the reader to [4] for a general theory of Banach lattices. We have, then, that $\mathscr{M}^{\mathrm{U}}=\mathscr{M}$, and $E^{c}\left(S_{1}\right)$ is the unique band projection on $\mathscr{M}^{\mathrm{I}}$. Since $E(S)$ is a contractive projection, a classical theorem (see [4], page 160 , Theorem 2) implies that $E(S) E^{c}\left(S_{1}\right)=$ $E^{c}\left(S_{1}\right)$. Combining these we finally obtain $E(S)=E^{c}\left(S_{1}\right)$. This finishes the proof.

Corollary 2 (Douglas-Pearcy [2]). If $A$ is a normal operator on the separable Hilbert space $H$ with spectral measure $\left\{E_{\lambda}\right\}$, then $\mathscr{H}$ is hyperinvariant for $A$ iff $\mathscr{M}=E(S) H$ for some Borel set $S$ of $\mathbb{C}$.

Proof. By the spectral theorem, we may assume that $A=M_{\phi}$ on $L^{2}(X, \mu)$ for some measurable space $(X, \mu)$. Also, since $H$ is separable by hypothesis, so is $L^{2}(X, \mu)$. The result now follows from Theorem 1 . 
Note. This result was proved by Douglas and Pearcy [2] using facts from the theory of von Neumann algebras. Our proof seems to be more elementary and comes almost directly from the spectral theorem.

\section{ACKNOWLEDGMENT}

The author wishes to thank the referee for his valuable advice and meticulous help.

\section{REFERENCES}

1. J. Diestel and J. J. Uhl, Jr., Vector measures, Amer. Math. Soc., Providence, R. I., 1977.

2. R. G. Douglas and C. Pearcy, On a topology for invariant subspaces, J. Funct. Anal. 2 (1968), 323-341.

3. N. Dunford, Spectral operators, Pacific J. Math. 4 (1954), 321-354.

4. H. E. Lacey, The isometric theory of classical Banach spaces, Springer-Verlag, Berlin, Heidelberg, New York, 1974.

5. H. Radjavi and P. Rosenthal, Hyperinvariant subspaces for spectral and n-normal operators, Acta Sci. Math. (Szeged) 32 (1971), 121-126.

6. __ Invariant subspaces, Springer-Verlag, Berlin, Heidelberg, New York, 1973.

Department of Mathematics, Nankai University, Tianjin City, People's Republic of CHINA 